hep-th/0604070

\title{
On Euclidean and Noetherian Entropies in AdS Space
}

\author{
Suvankar Dutta and Rajesh Gopakumar \\ Harish-Chandra Research Institute \\ Chhatnag Road, Jhusi, Allahabad 211019, INDIA \\ E-mail: suvankar@mri.ernet.in, gopakumr@mri.ernet.in
}

\begin{abstract}
We examine the Euclidean action approach, as well as that of Wald, to the entropy of black holes in asymptotically $A d S$ spaces. From the point of view of holography these two approaches are somewhat complementary in spirit and it is not obvious why they should give the same answer in the presence of arbitrary higher derivative gravity corrections. For the case of the $A d S_{5}$ Schwarzschild black hole, we explicitly study the leading correction to the Bekenstein-Hawking entropy in the presence of a variety of higher derivative corrections studied in the literature, including the Type IIB $R^{4}$ term. We find a non-trivial agreement between the two approaches in every case. Finally, we give a general way of understanding the equivalence of these two approaches.
\end{abstract}




\section{Contents}

1 Introduction $\quad 2$

2 A Brief Overview of the Euclidean Method 5

3 A Brief Overview of Wald's Approach $\quad 9$

3.1 Construction of Noether charges . . . . . . . . . . . . . . . . 9 9

3.2 Hamiltonians and Noether Charges . . . . . . . . . . . . . . . 11

3.3 Entropy as Noether charge . . . . . . . . . . . . . . . . . . . . 12

4 Correction to the entropy from higher derivative terms 13

4.1 The Gauss-Bonnet term . . . . . . . . . . . . . . . . . . . 13

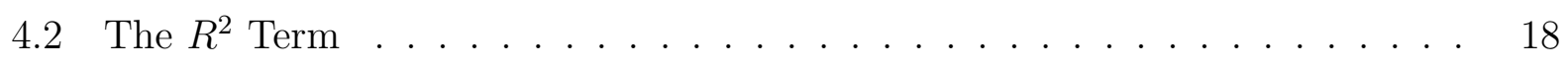

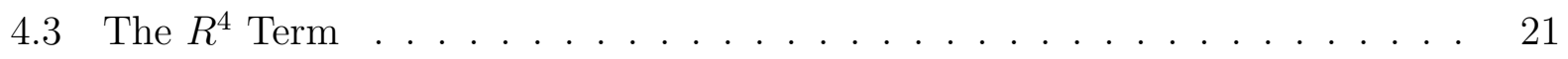

5 Wald's Approach Vs. Euclidean Approach in Asymptotically AdS Spacetime 26

5.1 Mass in asymptotically AdS spacetime . . . . . . . . . . . . . . 27

5.2 Relating the Wald and Euclidean approaches . . . . . . . . . . . . . 28

6 Conclusions $\quad 30$

$\begin{array}{ll}\text { A Calculation of Energy } & 31\end{array}$

B Relating the Noetherian and Euclidean definitions of Mass 32

\section{Introduction}

The Bekenstein-Hawking entropy of black holes was one of the first clues to the holographic nature of gravity. It indicated that any microscopic accounting of this entropy would entail that the underlying degrees of freedom are those of a local theory in one lower dimension. The AdS/CFT conjecture $[1,2,3]$ has been a remarkable realisation of this idea, giving a detailed dictionary between a theory of gravity and a gauge theory in one lower dimension.

Nevertheless, holography remains quite mysterious as a dynamical property of a theory with general covariance. To gain a better understanding of the mechanism giving rise to holography in theories of gravity, it seems worthwhile to go back to a closer examination 


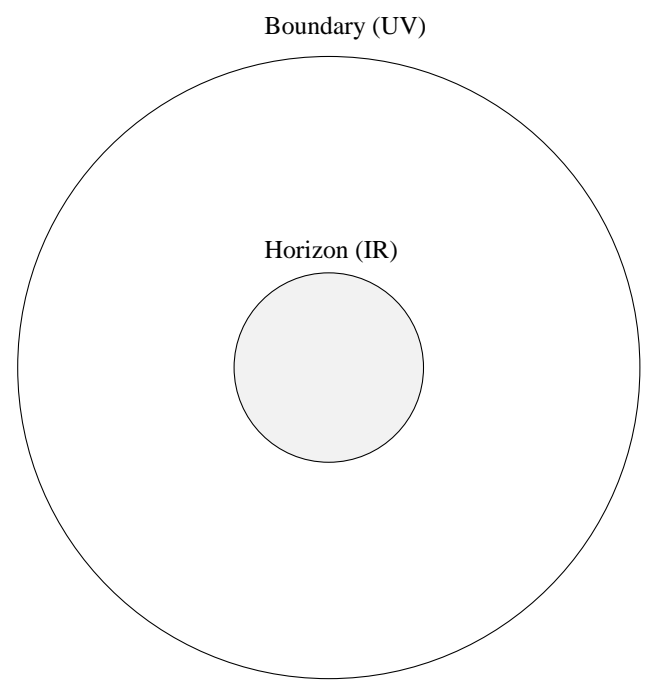

Figure 1: RG flow between two holographic descriptions

of the entropy of black holes. In this context, the Noetherian (or Wald's) approach to the calculation of entropy in theories of gravity, with arbitrary higher derivative corrections to the Einstein-Hilbert action, is particularly relevant [4]. For it not only provides a concrete method to calculate systematic corrections to the area law entropy, but also gives the answer in a form which is holographic in spirit. The entropy is given in terms of local quantities evaluated on the so-called bifurcate horizon, which is a special codimension two surface in the black hole spacetime.

We might therefore imagine a direct relation of this entropy to the holographic description afforded in asymptotically anti-de Sitter spacetimes. In this case, the entropy of a black hole in AdS space is related to the thermodynamic entropy of the boundary gauge theory at a finite temperature (which is the same as the Hawking temperature of the black hole). Thus there are two apparently different holographic descriptions of the entropy. One in terms of the horizon and the other in terms of the boundary gauge theory. In a rough sense, the latter is the UV description from the microscopic (gauge theory) point of view, while the former is the IR description, more appropriate from the coarse grained gravity point of view. One might therefore expect some kind of RG flow to relate the two. One of the original motivations behind the present work was to try and make more precise this kind of relation between these two descriptions. To that end, recall that the AdS/CFT dictionary is very naturally phrased 
in terms of a Euclidean functional integral relation between bulk and boundary quantities ${ }^{1}$. In particular, the free energy (or entropy) of the Euclidean thermal field theory is the same as the free energy (or entropy) of the gravity configuration as evaluated by the Euclidean gravitational action. Therefore it is essential to understand the relation, in the presence of higher derivative corrections to the Einstein action, between the Euclidean computation of black hole entropy and Wald's prescription. This would be a first step in relating the holographic gauge description to the holographic Wald description.

If both the Euclidean and Noetherian approaches to entropy are to be sensible prescriptions for obtaining the entropy in a gravity theory in AdS, then they ought to give the same answer $^{2}$. However, this is not immediately apparent. For one, the original derivation by Wald explicitly made use of the asymptotically flat nature of the space time. No complete proof seems to have been given yet for the similar result in asymptotically AdS space ${ }^{3}$. On the other hand, the Euclidean action prescription in asymptotically AdS space (going back to Hawking and Page [5]) makes use of a nontrivial background subtraction procedure. We will review this procedure in the next section. For now, we merely remark that due to the fact that there is a relation between the sizes of the thermal circles of the background AdS and the black hole, this is not just a matter of removing an infinite additive constant, but is crucial to getting the correct finite answer.

To illustrate the non-trivial nature of the agreement between these two approaches we present some explicit computations of the leading corrections to the entropy of a five dimensional $A d S$ Schwarzschild black hole in the presence of a variety of higher derivative gravitational corrections. The terms we consider are those that arise as $\alpha^{\prime}$ corrections in string theory. The first case is the addition of a Gauss-Bonnet term to the Einstein Hilbert action. This has been studied in detail in the literature (see for example [9] and references therein) and we use it as a warmup example. The next case is of a genuinely higher derivative term $R_{\mu \nu \rho \sigma} R^{\mu \nu \rho \sigma}$. This was studied in $[10,11]$ and there is some confusion regarding this term. In [10], disagreement was claimed between the Euclidean and Wald's expressions

\footnotetext{
${ }^{1}$ On the bulk side the Euclidean functional integral is to be understood only in a semiclassical sense which we can continue to use in the presence of higher derivative corrections, i.e. in an expansion about the large $N$, strong 'tHooft coupling limit.

${ }^{2}$ In the case of $A d S_{3}$, the Euclidean approach was combined with that of Wald for studying the effect of Chern-Simons terms [6]. See also [7,8].

${ }^{3}$ We have been informed by K. Skenderis (private communication) that the boundary counterterm method employed in [14] can be generalised to the higher derivative case and be used to demonstrate Wald's result for asymptotically locally AdS spaces. The boundary counterterm procedure then also provides a link to the Euclidean approach.
} 
even to leading order in $\alpha^{\prime}$. [11] obtained a different result which actually agrees with Wald's answer (though there is an unnecessary remark here that the agreement is only for large mass black holes). We find complete agreement between the Euclidean and the Wald approaches, to this order. Finally, we consider the leading $R^{4}$ correction that arises in Type IIB supergravity, which plays an important role in the AdS/CFT context. For this term, the authors of [12] had already studied the correction to the entropy of the planar AdS Schwarzschild black hole in the Euclidean approach. Here we apply Wald's prescription to this term and find exactly the same correction as obtained by [12]. In fact, we also find agreement in the case of the usual AdS Schwarzschild black hole (i.e. not just in the large mass or planar limit). As we will see, in all cases, the agreement is not at all manifest from the prescriptions themselves.

Prompted by this agreement and motivated by our desire to directly connect the two approaches, we present an argument to understand this equivalence. We use the Noetherian definition of mass in asymptotically AdS spaces given in $[13]^{4}$ and show that the subtraction scheme used to define the entropy in the Euclidean approach arises quite naturally in the Wald approach. For simplicity, we restrict to static cases.

The organisation of this paper is as follows. In the next two sections we give a quick overview of the Euclidean and the Wald methods respectively. Then we present in Section 4 , the computations for the individual cases of higher derivative corrections. In section 5 , we give the argument for why the two methods should give the same results. The last section carries some of the conclusions. In a first appendix, we illustrate the Hamiltonian definition of mass in AdS with a specific example. In the second appendix, we argue that the the Euclidean definition of mass must agree with the Hamiltonian one.

\section{A Brief Overview of the Euclidean Method}

In this section and the next we will give a quick overview of the two different approaches to calculating the entropy of black holes in asymptotically Anti-de Sitter space. We start with the Euclidean approach ${ }^{5}$ where a precise form of subtraction is important in getting the correct energy and entropy of AdS black holes. We will illustrate this procedure through the case of the five dimensional AdS-Schwarzschild black hole.

\footnotetext{
${ }^{4}$ See relatedly, [14] where things are worked out for the two derivative Lagrangian. [15, 16] attempt to generalise some of the considerations of [13] to a class of higher derivative Lagrangians.

${ }^{5}$ A somewhat different Euclidean prescription to compute the black hole entropy is given in [17]
} 
The Euclidean $A d S_{5}$ metric is given by,

$$
d s_{A d S}^{2}=\left(1+\frac{r^{2}}{b^{2}}\right) d \tau^{2}+\frac{d r^{2}}{\left(1+\frac{r^{2}}{b^{2}}\right)}+r^{2} d \Omega_{3}^{2} .
$$

Here $b$ is the radius of $A d S$ space. In these coordinates the Euclidean time $\tau$ of the anti-de Sitter space time can be taken to be periodic with arbitrary period $\beta^{\prime}$. This is then the Euclidean geometry describing thermal anti-de Sitter space, with inverse temperature $\beta^{\prime}$.

In these same coordinates, the Euclidean $A d S_{5}$ Schwarzschild metric is given by,

$$
d s_{B H}^{2}=\left(1+\frac{r^{2}}{b^{2}}-\frac{\omega M}{r^{2}}\right) d \tau^{2}+\frac{d r^{2}}{\left(1+\frac{r^{2}}{b^{2}}-\frac{\omega M}{r^{2}}\right)}+r^{2} d \Omega_{3}^{2} .
$$

Where,

$$
\omega=\frac{16 \pi G_{5}}{3 V_{3}}
$$

with $V_{3}$ being the volume of the unit three sphere. This space time has a horizon at $r=r_{+}$, given by,

$$
1+\frac{r_{+}^{2}}{b^{2}}-\frac{\omega M}{r_{+}^{2}}=0 .
$$

The apparent singularity at $r=r_{+}$is just like the singularity at the origin in the polar coordinate system and can be removed by regarding $\tau$ as an angular variable with period,

$$
\beta=\frac{2 \pi b^{2} r_{+}}{2 r_{+}^{2}+b^{2}} \equiv \beta_{0}
$$

Therefore, unlike pure AdS space where the time coordinate can have an arbitrary period, that of the AdS black hole has a well defined periodicity, for a given mass. This is identified with the (inverse) Hawking temperature of the black hole.

Both these metrics arise as solutions to the equation of motion

$$
R_{\mu \nu}=-\frac{4}{b^{2}} g_{\mu \nu}
$$

which follows from the Einstein Hilbert action.

$$
I_{E H}=-\frac{1}{16 \pi G_{5}} \int d^{5} x \sqrt{-g}\left(R+\frac{12}{b^{2}}\right) .
$$

In what follows we will consider higher derivative corrections to the Einstein-Hilbert Lagrangian. These will lead, in particular, to systematic corrections to the black hole metric, 
Eq. 2.2, which we can compute in a perturbative expansion in the coefficients of the additional terms. We are interested in the corrections to the thermodynamics of the black hole spacetime from these terms.

Therefore, let us first discuss the thermodynamics of black holes in the Euclidean framework as generally prescribed by Gibbons and Hawking [18]. The canonical partition function is defined by a functional integral over metrics with the Euclidean time coordinate $\tau$ identified with period $\beta$, defined above.

$$
Z=\int[\mathcal{D} g] e^{-I_{E}}
$$

$I_{E}$ is the Euclidean action which could have the Einstein-Hilbert piece as well as higher derivative corrections. In the semi-classical limit that we are considering, the dominant contribution to the path integral comes from classical solutions to the equations of motion. In this case,

$$
\ln Z=-I_{E}^{c l} .
$$

Thus the action $I_{E}^{c l}$, evaluated on solutions, is proportional to the free energy. Therefore the energy (or mass) of the black hole is given by,

$$
E=-\frac{\partial}{\partial \beta} \ln Z=\frac{\partial I_{E}^{c l}}{\partial \beta}
$$

and the entropy of the black hole is given by

$$
S=\beta E-I_{E}^{c l} .
$$

For asymptotically AdS black holes, the prescription needs to be modified. This is suggested by the fact that if we calculate the action $I_{E}^{c l}$ on a solution to the equation of motion, it turns out to be infinite. So we need to specify a subtraction procedure which will give sensible answers. To get the right results one subtracts the contribution of global AdS after a suitable regularisation. However, one has to be careful in doing this [5]. There are two points to be kept in mind: (i) For the black hole spacetime we evaluate the action integral only in the region $r_{+} \leq r \leq \tilde{R}$ where $\tilde{R}$ is an IR cutoff on the spacetime and $r_{+}$ is the location of the horizon. Whereas in the AdS space time the region of integration is $0 \leq r \leq \tilde{R}$. (ii) The crucial point, however, is that we do not take the black hole spacetime and the reference AdS spacetime as having the same periodicity in the time direction. The black hole spacetime has a fixed periodicity $\beta$ (for instance given by (2.8) in the case of the AdS-Schwarzschild metric). One adjusts the period $\beta^{\prime}$ of the globally AdS spacetime such that the geometry at the hypersurface $r=\tilde{R}$ is the same in both cases [5], i.e,

$$
\beta\left[g_{\tau \tau}^{B H}(r=\tilde{R})\right]^{1 / 2}=\beta^{\prime}\left[g_{\tau \tau}^{A d S}(r=\tilde{R})\right]^{1 / 2} .
$$


Through this relation, the periodicity of the reference AdS spacetime depends on the parameters (such as the mass etc.) of the black hole spacetime.

Let us illustrate how this regularisation, specified above, works in the simplest case of the Einstein-Hilbert action [5]. This will also show how the subtraction does not just remove the divergent piece but is also important in getting the finite contributions to the mass and entropy correct.

Evaluating the Einstein-Hilbert action on the solution Eq.2.2, we get,

$$
I_{B H}=\frac{2 \beta}{3 \omega b^{2}}\left(\tilde{R}^{4}-r_{+}^{4}\right)
$$

As prescribed, the radial integration has been carried out from $r_{+}$to $\tilde{R}$, with $\tilde{R} \rightarrow \infty$. We can then calculate the mass using Eq. 2.10 together with the relations Eqs.2.4 and 2.5. We obtain 6

$$
\frac{\partial}{\partial \beta} I_{B H}=\frac{2}{3 \omega b^{2}} \tilde{R}^{4}+\frac{2 M}{3}-\frac{2}{3 \omega b^{2}} \frac{r_{+}^{2}\left(2 r_{+}^{2}+b^{2}\right)^{2}}{\left(b^{2}-2 r_{+}^{2}\right)} .
$$

The first term is the divergent piece while the rest are finite. We can also naively calculate the entropy using Eq. 2.11.

$$
\begin{aligned}
\tilde{S}= & \beta \frac{\partial}{\partial \beta} I_{B H}-I_{B H} \\
& =\left(\frac{V_{3} r_{+}^{3}}{4 G_{5}}\right) \frac{4 r_{+}^{2}}{2 r_{+}^{2}-b^{2}}
\end{aligned}
$$

Note that the entropy as calculated with the above cutoff, gives a finite answer. The divergent pieces have canceled out. But this finite piece is not the correct area entropy which one expects for the Einstein-Hilbert action. As we will see, we obtain the right answer only after the prescribed subtraction of the reference AdS geometry.

Similarly one can evaluate the action on the (regularised) AdS spacetime

$$
I_{A d S}=\frac{2 \beta^{\prime}}{3 \omega b^{2}} \tilde{R}^{4}
$$

Here the radial integration has been done from zero to $\tilde{R}$. $\beta$ and $\beta^{\prime}$ are related by Eq. 2.12. For large $\tilde{R}$ we get,

$$
\beta^{\prime}=\beta\left(1-\frac{\omega M b^{2}}{2 \tilde{R}^{4}},\right)
$$

\footnotetext{
${ }^{6}$ Here we have chosen to write the expression in a particular combination which will be used later. The main point to notice is that the finite part of $\partial I / \partial \beta$ does not give the mass of the black hole.
} 
where $\omega M$ is a function of $r_{+}$given by Eq. 2.4. We then find

$$
\frac{\partial}{\partial \beta} I_{A d S}=\frac{2}{3 \omega b^{2}} \tilde{R}^{4}-\frac{M}{3}-\frac{2}{3 \omega b^{2}} \frac{r_{+}^{2}\left(2 r_{+}^{2}+b^{2}\right)^{2}}{\left(b^{2}-2 r_{+}^{2}\right)} .
$$

Subtracting Eq.2.18 from Eq.2.14 we find that the energy of the black hole is exactly M! Similarly, for the entropy, we evaluate

$$
\beta \frac{\partial}{\partial \beta} I_{A d S}-I_{A d S}=-\frac{4 \pi b^{2}}{3 \omega b^{2}} \frac{r_{+}^{3}\left(2 r_{+}^{2}+b^{2}\right)}{b^{2}-2 r_{+}^{2}} .
$$

We need to subtract Eq. 2.19 from Eq. 2.15 to get the entropy

$$
S=\left(\frac{V_{3} r_{+}^{3}}{4 G_{5}}\right)=\frac{A_{B H}}{4 G_{5}} .
$$

Thus we get the expected answer from the nontrivial interplay between the actions evaluated on the AdS Schwarzschild geometry and the background AdS spacetime.

\section{A Brief Overview of Wald's Approach}

The essence of Wald's approach consists of three steps. The first is to give a general expression for Noether currents and charges corresponding to arbitrary diffeomorphisms. The next step is to use this to construct Hamiltonians corresponding to these Noether charges. In special cases, these Hamiltonians are related to the usual conserved charges such as mass, angular momentum etc. and are given by surface integrals at infinity on a Cauchy surface. Finally, in the case of certain killing vectors such as for time translation invariance one can relate the variation of the Hamiltonian (corresponding to say, energy) to that of the Noether charge evaluated on the bifurcate horizon. This relation can then be interpreted as the first law of black hole thermodynamics with the latter quantity identified as being proportional to the entropy. We will now give a quick review of these steps, referring the reader to the original papers $[4,19]$ for more details.

\subsection{Construction of Noether charges}

Let $\mathbf{L}$ be a diffeomorphism invariant Lagrangian, in $n$ spacetime dimensions built out of the metric and other fields, collectively denoted by $\psi^{7}$. Under any arbitrary field variation $\delta \psi$

\footnotetext{
${ }^{7}$ We will follow Wald's convention of viewing the Lagrangian as a top form rather than a scalar density. Similarly, other tensor densities will also be dualised and viewed as appropriate forms. To distinguish these from the usual densities, we will denote the forms in boldface.
} 
the Lagrangian varies as,

$$
\delta \mathbf{L}=\mathbf{E}(\psi) \delta \psi+d \boldsymbol{\Theta}(\delta \psi) .
$$

The classical equations of motion are given by $\mathbf{E}=0$. The second term is a boundary term which can depend on both $\delta \psi$ and its derivatives. The notation is abbreviated here so that a sum over the tensor indices, for instance for $\delta \psi$, is understood.

For instance, for a generally covariant Lagrangian which is a function of the metric and different powers of the Riemann tensor, but not covariant derivatives of the Riemann tensor, $\Theta$ can be chosen to be of the form

$$
\Theta^{a}(\delta g)=2 E_{R}^{a b c d} \nabla_{d} \delta g_{b c}-2 \nabla_{d} E_{R}^{a b c d} \delta g_{b c},
$$

where

$$
E_{R}^{a b c d}=\frac{\partial L}{\partial R_{a b c d}} .
$$

We will actually be interested in diffeomorphisms, where the field variations are given by the Lie derivative $\delta \psi=\mathcal{L}_{\xi} \psi$ ( $\xi^{a}$ is the infinitesimal generator of a diffeomorphism). The resulting variation of a covariant Lagrangian is then a total derivative.

$$
\delta \mathbf{L}=\mathcal{L}_{\xi} \mathbf{L}=d(\xi \cdot \mathbf{L}) .
$$

Here the '.' denotes the usual contraction of a vector field with a form.

Thus, in this case we can define a current

$$
J_{\xi}^{a}=\Theta^{a}\left(\mathcal{L}_{\xi} \psi\right)-\xi^{a} L .
$$

Or equivalently in terms of $(n-1)$ forms, in the dualised notation

$$
\mathbf{J}_{\xi}=\boldsymbol{\Theta}\left(\mathcal{L}_{\xi} \psi\right)-\xi \cdot \mathbf{L} .
$$

It satisfies

$$
d \mathbf{J}_{\xi}=-\mathbf{E} \mathcal{L}_{\xi} \psi .
$$

So $J_{\xi}^{a}$ is a Noether current which is conserved for any $\xi$ when the equations of motion are satisfied.

Now, for any conserved $J_{\xi}$ there (locally) exists a $(n-2)$ form "Noether Charge" $\mathbf{Q}_{\xi}(\psi)$ constructed out of fields $\psi$ and $\xi^{a}[20]$, such that whenever $\psi$ satisfies the equation of motion, we have,

$$
\mathbf{J}_{\xi}=d \mathbf{Q}_{\xi}(\psi) .
$$

In what follows we will always take $\psi$ to be a solution of the equations of motion. 


\subsection{Hamiltonians and Noether Charges}

As mentioned above, the second step is to relate these Noether charges to Hamiltonians generating the diffeomorphisms $\xi$ (see also [21]). The essential point here is that the boundary term $\Theta(\delta \psi)$ acts as a "symplectic potential" which enables one to define a symplectic form on the phase space of field configurations. Considering two arbitrary variations $\delta_{1} \psi$ and $\delta_{2} \psi$, we can define a symplectic current $\omega$ by

$$
\omega\left(\delta_{1} \psi, \delta_{2} \psi\right)=\delta_{2} \Theta\left(\delta_{1} \psi\right)-\delta_{1} \Theta\left(\delta_{2} \psi\right)
$$

The symplectic form (on the space of variations $\delta \psi$ ) itself is then defined by an integral over a Cauchy surface $\Sigma$

$$
\Omega\left(\delta_{1} \psi, \delta_{2} \psi\right)=\int_{\Sigma} \omega\left(\delta_{1} \psi, \delta_{2} \psi\right)
$$

So now an arbitrary variation $\delta \psi$ of the Noether current can be re-expressed in terms of the symplectic form

$$
\begin{aligned}
\delta \mathbf{J}_{\xi} & =\delta \boldsymbol{\Theta}\left(\mathcal{L}_{\xi} \psi\right)-\xi \cdot \delta \mathbf{L} \\
& =\omega\left(\delta \psi, \mathcal{L}_{\xi} \psi\right)+d(\xi \cdot \boldsymbol{\Theta}(\delta \psi)) .
\end{aligned}
$$

In the second equality, we have used the fact that $\psi$ is a solution to the equations of motion as well as an identity for Lie derivatives. Integrating Eq.3.11 over the Cauchy surface and since the Hamiltonian for a vector field $\xi$ is defined via

$$
\delta H_{\xi}=\Omega\left(\delta \psi, \mathcal{L}_{\xi} \psi\right)
$$

we have

$$
\begin{aligned}
\delta H_{\xi} & =\int_{\Sigma} \delta \mathbf{J}_{\psi}-\int_{\partial \Sigma} \xi \cdot \boldsymbol{\Theta}(\delta \psi) \\
& =\int_{\partial \Sigma}\left(\delta \mathbf{Q}_{\xi}-\xi \cdot \boldsymbol{\Theta}(\delta \psi)\right) .
\end{aligned}
$$

In the second equality we have evaluated the Hamiltonian on-shell, where $\mathbf{J}_{\psi}=d \mathbf{Q}_{\psi}$. Furthermore, if the boundary term arises from the variation of a boundary term in the action,

$$
\Theta(\delta \psi)=\delta \mathbf{B}
$$

then the above equation can be integrated to obtain a Hamiltonian $H_{\xi}$. If $\Sigma$ has only an asymptotic boundary at infinity then the above Hamiltonian is expressed purely as a surface integral at infinity. In the asymptotically flat case the Hamiltonian corresponding to an asymptotic time translation vector field was shown to be equal to the ADM mass in the case of the Einstein-Hilbert Lagrangian. We will discuss the asymptotically AdS case later. 


\subsection{Entropy as Noether charge}

We can now apply these considerations to killing vector fields. In particular, we will specialise to killing vector fields $\xi_{H}^{a}$ which are null on the codimension one horizon and vanish on the codimension two surface called the bifurcate horizon $\mathcal{H}$ of a black hole spacetime.

To obtain the first law of black hole thermodynamics, we first consider a variation $\delta \psi$ in Eq.3.11, which satisfies the linearised equations of motion. Since we are considering a Killing vector field, for which $\mathcal{L}_{\xi} \psi=0$, we also have $\omega\left(\delta \psi, \mathcal{L}_{\xi} \psi\right)=0$. We use this and integrate both sides of Eq. 3.11 over a spatial hypersurface $C$ of the black hole spacetime which has $\mathcal{H}$ as its interior boundary (in addition to asymptotic infinity). Since $\delta \psi$ satisfies the equations of motion, we have $\delta \mathbf{J}=d(\delta \mathbf{Q})$. The integrand on both sides reduce to total derivatives. The integral thus gets contributions from only the interior boundary and from infinity.

For instance, we might take

$$
\xi_{H}^{a}=\frac{\partial}{\partial t}
$$

in a static black hole background spacetime. In such a case, using Eq.3.13, the outer boundary contribution gives exactly the change in the energy or mass. Thus we get a relation of the form

$$
\delta \int_{\mathcal{H}} d S_{a b} \sqrt{-g} Q^{a b}=\delta \mathcal{E} .
$$

It turns out to be consistent to make an identification with the entropy $S$ via

$$
\frac{\kappa}{2 \pi} \delta S=\delta \int_{\mathcal{H}} d S_{a b} \sqrt{-g} Q^{a b}
$$

where $\kappa$ is the surface gravity (which is constant over $\mathcal{H}$ ) and is proportional to the temperature. Therefore Eq.3.16 is the first law of black hole thermodynamics,

$$
\frac{1}{\beta} \delta S=\delta \mathcal{E}
$$

Moreover, from the identification with the Noether charge in Eq. 3.17, Iyer and Wald [19] also found a simple expression for the entropy. Using the definition of the Noether charge and with the Noether current given by Eq.3.5, they expressed the entropy in terms of the Lagrangian as

$$
S=-2 \pi \int_{\mathcal{H}} \frac{\delta L}{\delta R_{a b c d}} \epsilon_{a b} \epsilon_{c d}
$$

where $\epsilon_{a b}$ is the binormal to the surface $\mathcal{H}$. In the special case where the Lagrangian does not depend on derivatives of the Riemann tensor, we have

$$
S=-2 \pi \int_{\mathcal{H}} \frac{\partial L}{\partial R_{a b c d}} \epsilon_{a b} \epsilon_{c d} .
$$


It is this expression that we will have occasion to use in the next section in comparing with the Euclidean answers.

\section{Correction to the entropy from higher derivative terms}

In this section we will consider some explicit examples of higher derivative corrections to the Einstein-Hilbert action (with negative cosmological constant). The examples are all ones that arise as $\alpha^{\prime}$ corrections in various string theory effective actions. They include a) the Gauss-Bonnet term, b) $R_{\mu \nu \rho \sigma} R^{\mu \nu \rho \sigma}$ term and c) the Type IIB $R^{4}$ or equivalently $(W e y l)^{4}$ term. In all these cases we calculate the leading correction to the entropy of the black hole geometry which is asymptotically AdS. Keeping in mind the application to holography and the AdS/CFT conjecture, we restrict ourselves to the case of five dimensional spacetime.

The calculations are done using both the Euclidean approach as well as using Wald's formula. In the Euclidean approach this requires one to correct the leading AdS-Schwarzschild metric and then evaluate the action and thus entropy according to the prescription outlined in Sec.2. Similarly, in using Wald's formula, we need to evaluate the corrections to the area law entropy coming from the additional contributions to Eq.3.19. We find agreement between the two separate calculations in every case, unlike the claims in the literature to the contrary. As mentioned in the Introduction, the agreement between these very different seeming modes of calculation is quite surprising. These explicit checks therefore gives one mutually reinforcing confidence in both approaches.

\subsection{The Gauss-Bonnet term}

The Gauss-Bonnet term is a very natural correction term to the Einstein action. It has the feature that despite being built out of terms which individually give rise to higher than two derivative equations of motion, the full equation of motion actually has only two derivatives. This term arises in both the heterotic and bosonic string theory low energy effective actions, after a suitable field redefinition.

In this case exact black hole solutions are known (see for e.g. [9]). However, in keeping with the other cases where we do not have this luxury, we will work only to leading order in this term. The action containing the Gauss-Bonnet term is

$$
I=-\frac{1}{16 \pi G_{5}} \int d^{5} x \sqrt{-g}\left(R+\frac{12}{b^{2}}\right)-\frac{\alpha^{\prime} / 4}{16 \pi G_{5}} \int d^{5} x \sqrt{-g} L_{G . B} .
$$


where,

$$
L_{G . B .}=R_{\mu \nu \rho \sigma} R^{\mu \nu \rho \sigma}-4 R_{\mu \nu} R^{\mu \nu}+R^{2} .
$$

The particular coefficient $\alpha^{\prime} / 4$ is chosen so that $I$ matches with the low energy effective action of heterotic string theory.

The equation of motion for this action is,

$$
R_{\mu \nu}-\frac{1}{2} R g_{\mu \nu}-\frac{6}{b^{2}} g_{\mu \nu}=\frac{\alpha^{\prime}}{8} g_{\mu \nu} L_{G . B .}-\frac{\alpha^{\prime}}{2}\left(R_{\mu \rho \sigma \delta} R_{\nu}^{\rho \sigma \delta}-2 R^{\rho \sigma} R_{\mu \rho \nu \sigma}-2 R_{\mu}^{\rho} R_{\nu \rho}+R R_{\mu \nu}\right),
$$

from which we get,

$$
R=-\frac{20}{b^{2}}-\frac{\alpha^{\prime}}{12} L_{G \cdot B}
$$

\section{- Correction to the Metric}

We are interested in treating the Gauss-Bonnet term as a perturbation and finding the leading correction in $\alpha^{\prime}$ to the AdS Schwarzschild metric. We will therefore look for a solution of the spherically symmetric, static form

$$
d s^{2}=B(r) d \tau^{2}+A(r) d r^{2}+r^{2} d \Omega_{3}^{2}
$$

where,

$$
A(r)=A_{0}(r)\left(1+\alpha^{\prime} \mu(r)\right), \quad A_{0}(r)=\left(1+\frac{r^{2}}{b^{2}}-\frac{\omega M}{r^{2}}\right)^{-1}
$$

and

$$
B(r)=B_{0}(r)\left(1+\alpha^{\prime} \varepsilon(r)\right), \quad B_{0}(r)=\left(1+\frac{r^{2}}{b^{2}}-\frac{\omega M}{r^{2}}\right) .
$$

We can solve the equations of motion Eq.4.3 keeping in mind that we can use the unperturbed metric in evaluating the terms proportional to $\alpha^{\prime}$. We obtain

$$
\mu(r)=-A_{0}(r)\left(\frac{r^{2}}{2 b^{4}}+\frac{M^{2} \omega^{2}}{2 r^{6}}\right) ; \quad \varepsilon(r)=-\mu(r) .
$$

So that

$$
A(r)=B(r)^{-1}=\left(1+\frac{r^{2}}{b^{2}}-\frac{\omega M}{r^{2}}+\alpha^{\prime} \frac{r^{2}}{2 b^{4}}+\alpha^{\prime} \frac{\omega^{2} M^{2}}{2 r^{6}}\right)^{-1} .
$$

This solution matches with the solution found in $[9]^{8}$.

\footnotetext{
${ }^{8}$ The parameter $M$ which appears in this solution is not the mass in the presence of this correction. In comparing with [9], this has to be kept in mind.
} 


\section{- Correction to the Black Hole Temperature}

The Euclidean time direction has a periodicity $\beta$ fixed by requiring the geometry to be smooth at the horizon. For a spherically symmetric metric of the form in Eq. 4.5, the inverse temperature of the black hole is therefore

$$
\beta=\frac{4 \pi}{B^{\prime}\left(r_{+}\right)}
$$

where, $r_{+}$is now the (corrected) location of the horizon of the black hole, $g_{\tau \tau}\left(r_{+}\right)=0$.

$$
\omega M=r_{+}^{2}\left(1+\frac{r_{+}^{2}}{b^{2}}+\alpha^{\prime} \frac{r_{+}^{2}}{b^{4}}+\frac{\alpha^{\prime}}{2 r_{+}^{2}}+\frac{\alpha^{\prime}}{b^{2}}\right) .
$$

Using Eq.4.9 we get the corrected inverse temperature,

$$
\beta=\frac{2 \pi b^{2} r_{+}}{2 r_{+}^{2}+b^{2}}\left(1+\frac{\alpha^{\prime}}{r_{+}^{2}}\right)=\beta_{0}\left(1+\frac{\alpha^{\prime}}{r_{+}^{2}}\right) .
$$

\section{- Calculation of Entropy}

Let us write the black hole action 4.1 to be,

$$
I_{B H}=-\frac{1}{16 \pi G_{5}}\left(I_{0}+I_{1}\right)
$$

where,

$$
I_{0}=\int d^{5} x \sqrt{-g}\left(R+\frac{12}{b^{2}}\right), \quad \text { and } \quad I_{1}=\frac{\alpha^{\prime}}{4} \int d^{5} x \sqrt{-g} L_{G . B .} .
$$

In $I_{0}$ we substitute the value of $R$ for the perturbed solution. For this we use Eq.4.4. In $I_{1}$ we can use the unperturbed metric, to this order in $\alpha^{\prime}$. So that

$$
L_{G . B}=\frac{120}{b^{4}}+\frac{72 \omega^{2} M^{2}}{r^{8}} .
$$

We get,

$$
\begin{gathered}
I_{0}=\int d^{5} x \sqrt{-g}\left(\frac{-20}{b^{2}}+\frac{12}{b^{2}}-\alpha^{\prime} \frac{10}{b^{4}}-\alpha^{\prime} \frac{6 M^{2} \omega^{2}}{r^{8}}\right) \\
=-\int_{0}^{\beta} d t \int_{0}^{\tilde{R}} d r \int d \Omega_{3} r^{3}\left(\frac{8}{b^{2}}+\alpha^{\prime} \frac{10}{b^{4}}+\frac{6 \alpha^{\prime} M^{2} \omega^{2}}{r^{8}}\right) \\
=-\beta V_{3}\left(\tilde{R}^{4}-r_{+}^{4}\right)\left(\frac{2}{b^{2}}+\alpha^{\prime} \frac{5}{2 b^{4}}+\frac{3 \alpha^{\prime} M^{2} \omega^{2}}{2 \tilde{R}^{4} r_{+}^{4}}\right)
\end{gathered}
$$

where $V_{3}$ is volume of unit 3 sphere, $V_{3}=2 \pi^{2} . I_{1}$ can be evaluated using the unperturbed metric. So that

$$
I_{1}=\alpha^{\prime} \beta V_{3}\left(\tilde{R}^{4}-r_{+}^{4}\right)\left(\frac{15}{2 b^{4}}+\frac{9 M^{2} \omega^{2}}{2 \tilde{R}^{4} r_{+}^{4}}\right) .
$$


Hence $I_{B H}$ is given by,

$$
I_{B H}=\frac{\beta V_{3}\left(\tilde{R}^{4}-r_{+}^{4}\right)}{16 \pi G_{5}}\left(\frac{2}{b^{2}}-\alpha^{\prime} \frac{5}{b^{4}}-\alpha^{\prime} \frac{3 M^{2} \omega^{2}}{\tilde{R}^{4} r_{+}^{4}}\right) .
$$

The next step is to calculate the action of the background $A d S$ spacetime,

$$
I_{A d S}=-\frac{1}{16 \pi G_{5}}\left(J_{0}+J_{1}\right)
$$

where,

$$
J_{0}=\int_{0}^{\beta^{\prime}} d t \int_{0}^{\tilde{R}} d r r^{3} \int d \Omega_{3}\left(R+\frac{12}{b^{2}}\right) \quad \text { and } \quad J_{1}=\frac{\alpha^{\prime}}{4} \int_{0}^{\beta^{\prime}} d t \int_{0}^{\tilde{R}} d r r^{3} \int d \Omega_{3} L_{G B} .
$$

Here $\beta^{\prime}$ is the periodicity of the time direction of the AdS space time. These expressions are easily evaluated,

$$
J_{0}=-\beta^{\prime} V_{3} \tilde{R}^{4}\left(\frac{2}{b^{2}}+\frac{5 \alpha^{\prime}}{2 b^{4}}\right)
$$

and

$$
J_{1}=\alpha^{\prime} \frac{15 \beta^{\prime} V_{3} \tilde{R}^{4}}{2 b^{4}}
$$

To evaluate $J_{0}$, we have used the perturbed $A d S$ metric which is obtained from Eq.4.9 by setting $M=0$. As before $J_{1}$ is evaluated using the leading order solution. So $I_{A d S}$ is given by,

$$
I_{A d S}=\frac{\beta^{\prime} V_{3} \tilde{R}^{4}}{16 \pi G_{5}}\left(\frac{2}{b^{2}}-\alpha^{\prime} \frac{5}{b^{4}}\right) .
$$

The difference between the AdS-Schwarzschild action and the AdS action is

$$
\begin{aligned}
\Delta I= & I_{B H}-I_{A d S} \\
= & -\frac{V_{3}}{16 \pi G_{5}}\left[\frac{2}{b^{2}} \tilde{R}^{4}\left(\beta^{\prime}-\beta\right)+\frac{2}{b^{2}} \beta r_{+}^{4}\right. \\
& \left.+\alpha^{\prime}\left(\frac{3 \beta M^{2} \omega^{2}}{r_{+}^{4}}-\frac{5 \beta r_{+}^{4}}{b^{4}}-\frac{5}{b^{4}} \tilde{R}^{4}\left(\beta^{\prime}-\beta\right)\right)\right] .
\end{aligned}
$$

Since at the outer boundary hypersurface $r=\tilde{R}$, the geometry of the $A d S$-Schwarzschild spacetime and $A d S$ is the same, $\beta$ and $\beta^{\prime}$ are related by Eq.2.12,

$$
\beta^{\prime}\left(1+\frac{\tilde{R}^{2}}{b^{2}}+\alpha^{\prime} \frac{\tilde{R}^{2}}{2 b^{4}}\right)^{1 / 2}=\beta\left(1+\frac{\tilde{R}^{2}}{b^{2}}-\frac{\omega M}{\tilde{R}^{2}}+\alpha^{\prime} \frac{\tilde{R}^{2}}{2 b^{4}}+\alpha^{\prime} \frac{\omega^{2} M^{2}}{2 \tilde{R}^{6}}\right)^{1 / 2} .
$$

In other words,

$$
\beta^{\prime}=\beta\left(1-\frac{1}{2 \tilde{R}^{4}} \omega M b^{2}+\alpha^{\prime} \frac{\omega M}{4 \tilde{R}^{4}}\right) .
$$


Substituting $\beta^{\prime}$ in terms of $\beta$ in equation 4.23 we get,

$$
\Delta I=-\frac{V_{3}}{16 \pi G_{5}} \beta\left[\left(\frac{r_{+}^{4}}{b^{2}}-r_{+}^{2}\right)+\alpha^{\prime}\left(\frac{8 r_{+}^{2}}{b^{2}}+\frac{5}{2}\right)\right] .
$$

Using the relation Eq.4.26 one can calculate the energy of the black hole, and it comes out to be,

$$
\begin{aligned}
E & =\frac{\partial \Delta I}{\partial \beta} \\
& =\frac{3 V_{3} r_{+}^{2}}{16 \pi G_{5}}\left(1+\frac{r_{+}^{2}}{b^{2}}+\frac{\alpha^{\prime}}{2 r_{+}^{2}}\right) \\
& =M\left(1-\frac{\alpha^{\prime}}{b^{2}}\right)
\end{aligned}
$$

This relation between the energy and the parameter $M$ agrees in comparing with those of [9]. The entropy of the black hole is given by

$$
S=\beta \frac{\partial \Delta I}{\partial \beta}-\Delta I
$$

Using equations 4.26, 4.12 and 4.27 the final corrected Euclidean entropy of the black hole works out to be,

$$
S=\frac{V_{3} r_{+}^{3}}{4 G_{5}}\left(1+\alpha^{\prime} \frac{3}{r_{+}^{2}}\right)
$$

One can now compare with the entropy using Wald's formula. It is given for instance by $[22]$,

$$
S_{\text {Wald }}=\frac{4 \pi}{16 \pi G_{5}} \int_{\mathcal{H}} d^{3} x \sqrt{h}\left[1+\frac{\alpha^{\prime}}{2} \mathcal{R}(h)\right]
$$

where $h$ is the determinant of induced metric on the spherical horizon and $\mathcal{R}(h)=h^{i j} h^{k l} R_{i k j l}$. $\mathcal{R}$ can be evaluated using the unperturbed metric on the sphere

$$
\mathcal{R}=\frac{6}{r_{+}^{2}}
$$

So $S_{\text {Wald }}$ exactly matches with the Euclidean entropy. Note that $b^{2}$ does not appear in the entropy. 


\subsection{The $R^{2}$ Term}

The second example we consider involves a correction proportional to $R_{\mu \nu \rho \sigma} R^{\mu \nu \rho \sigma}$ (which we will call the $R^{2}$ term for convenience). This is the first term that one can add to the Einstein-Hilbert action which genuinely has higher derivatives (in contrast to the GaussBonnet term) while also changing the $A d S$ Schwarzschild solution nontrivially to leading order. This example has been also studied in [10][11]. In [10], a discrepancy was claimed between the Euclidean and Wald expressions for the entropy, even to leading order. Our results here agree with [11] who also found that the two methods actually yield the same result for the leading correction in $\alpha^{\prime}$ (but for arbitrary mass black holes).

We will take the action containing the $R^{2}$ correction to be

$$
I=-\frac{1}{16 \pi G_{5}} \int d^{5} x \sqrt{-g}\left[\left(R+\frac{12}{b^{2}}\right)+\frac{\alpha^{\prime}}{4} R_{\mu \nu \rho \sigma} R^{\mu \nu \rho \sigma}\right] .
$$

Here we have again chosen the coefficient of the higher derivative term to be $\alpha^{\prime} / 4$ as in section 4.1. The equation of motion is given by,

$$
\begin{aligned}
R_{\mu \nu}-\frac{R}{2} g_{\mu \nu}-\frac{6}{b^{2}} g_{\mu \nu}= & -\frac{\alpha^{\prime}}{2}\left[R_{\mu}^{\rho \sigma \delta} R_{\nu \rho \sigma \delta}+2 \square R_{\mu \nu}-\frac{1}{2}\left(\nabla_{\mu} \nabla_{\nu}+\nabla_{\nu} \nabla_{\mu}\right) R\right. \\
& \left.+2 R^{\rho \sigma} R_{\mu \rho \nu \sigma}-2 R_{\mu}^{\rho} R_{\nu \rho}\right]+\frac{\alpha^{\prime}}{8} g_{\mu \nu} R_{\alpha \gamma \rho \sigma} R^{\alpha \gamma \rho \sigma} .
\end{aligned}
$$

For the $A d S$ Schwarzschild metric, this leads to a correction at order $\alpha^{\prime}$. In the right hand side of Eq.4.32, we substitute the unperturbed metric to obtain

$$
R_{\mu \nu}+\frac{4}{b^{2}} g_{\mu \nu}=-\frac{\alpha^{\prime}}{2}\left[J_{\mu \nu}-\left(\frac{20}{3 b^{4}}+\frac{12 M^{2} \omega^{2}}{r^{8}}\right) g_{\mu \nu}\right] .
$$

Where $J_{\mu \nu}$ is given by,

$$
J_{\mu \nu}=R_{\mu}^{\rho \sigma \delta} R_{\nu \rho \sigma \delta}
$$

evaluated on the metric 2.2. We also record for later,

$$
R=-\frac{20}{b^{2}}-\alpha^{\prime}\left(\frac{10}{3 b^{2}}+\frac{6 M^{2} \omega^{2}}{r^{8}}\right)
$$

\section{- Correction to the equation of motion}

We solve the equation of motion with the spherically symmetric ansatz, Eq. 4.5,

$$
d s^{2}=B(r) d \tau^{2}+A(r) d r^{2}+r^{2} d \Omega_{3}^{2} .
$$


Using the parametrisation Eqs.4.6,4.7 and solving for the functions $\varepsilon(r)$ and $\mu(r)$ we find

$$
\varepsilon(r)=\frac{r^{2}}{6 b^{4} B_{0}}+\frac{M^{2} \omega^{2}}{2 r^{6} B_{0}}, \quad \mu(r)=-\varepsilon(r) .
$$

Hence,

$$
B(r)=A(r)^{-1}=1+\frac{r^{2}}{b^{2}}-\frac{\omega M}{r^{2}}+\alpha^{\prime}\left(\frac{r^{2}}{6 b^{4}}+\frac{M^{2} \omega^{2}}{2 r^{6}}\right) .
$$

- Correction to the Black Hole Temperature

Using Eq. 4.10 with $r_{+}$given by the equation,

$$
\omega M=r_{+}^{2}+\frac{r_{+}^{4}}{b^{2}}+\frac{\alpha^{\prime}}{2}\left(1+\frac{2 r_{+}^{2}}{b^{2}}+\frac{4 r_{+}^{4}}{3 b^{4}}\right)
$$

the inverse temperature $\beta$ comes out to be,

$$
\beta=\frac{2 \pi b^{2} r_{+}}{2 r_{+}^{2}+b^{2}}\left[1+\frac{\alpha^{\prime}}{3 b^{2}} \frac{2 r_{+}^{4}+3 b^{4}+6 b^{2} r_{+}^{2}}{r_{+}^{2}\left(2 r_{+}^{2}+b^{2}\right)}\right]=\beta_{0}\left[1+\frac{\alpha^{\prime}}{3 b^{2}} \frac{2 r_{+}^{4}+3 b^{4}+6 b^{2} r_{+}^{2}}{r_{+}^{2}\left(2 r_{+}^{2}+b^{2}\right)}\right] .
$$

\section{- Calculation of Entropy}

The action 4.31 can be evaluated to be

$$
I_{B H}=-\frac{1}{16 \pi G_{5}}\left(I_{0}+I_{1}\right) .
$$

Here $I_{0}$ is evaluated using the perturbed solution

$$
\begin{aligned}
I_{0}= & \int d^{5} x \sqrt{-g}\left(R+\frac{12}{b^{2}}\right) \\
= & -\frac{2 V_{3} \beta}{b^{2}}\left(\tilde{R}^{4}-r_{+}^{4}\right)-\alpha^{\prime} \frac{5 V_{3} \beta}{6 b^{4}}\left(\tilde{R}^{4}-r_{+}^{4}\right) \\
& +\alpha^{\prime} \frac{3 M^{2} \omega^{2} V_{3} \beta}{2}\left(\frac{1}{\tilde{R}^{4}}-\frac{1}{r_{+}^{4}}\right) .
\end{aligned}
$$

To calculate $I_{1}$ we can use the unperturbed solution.

$$
\begin{aligned}
I_{1} & =\frac{\alpha^{\prime}}{4} \int d^{5} x \sqrt{-g} R_{\mu \nu \rho \sigma} R^{\mu \nu \rho \sigma} \\
& =\alpha^{\prime}\left[\frac{5 V_{3} \beta}{2 b^{4}}\left(\tilde{R}^{4}-r_{+}^{4}\right)+\frac{9 M^{2} \omega^{2} V_{3} \beta}{2} \frac{1}{r_{+}^{4}}\right] .
\end{aligned}
$$

So, finally we get,

$$
I_{B H}=-\frac{V_{3} \beta}{16 \pi G_{5}}\left[-\frac{2}{b^{2}}\left(\tilde{R}^{4}-r_{+}^{4}\right)+\alpha^{\prime} \frac{5}{3 b^{4}}\left(\tilde{R}^{4}-r_{+}^{4}\right)+\alpha^{\prime} \frac{3 M^{2} \omega^{2}}{r_{+}^{4}}\right] .
$$


Now let us similarly evaluate the background action for the $A d S$ spacetime,

$$
I_{A d S}=-\frac{1}{16 \pi G_{5}}\left(J_{0}+J_{1}\right)
$$

Where,

$$
J_{0}=-\frac{2}{b^{2}} \beta^{\prime} V_{3} \tilde{R}^{4}-\frac{5 \alpha^{\prime}}{6 b^{4}} \beta^{\prime} V_{3} \tilde{R}^{4}
$$

and,

$$
J_{1}=\frac{5 \alpha^{\prime}}{2 b^{4}} V_{3} \beta^{\prime} \tilde{R}^{4}
$$

So finally we get,

$$
I_{A d S}=-\frac{\beta^{\prime} V_{3}}{16 \pi G_{5}}\left[-\frac{2}{b^{2}} \tilde{R}^{4}+\alpha^{\prime} \frac{5}{3 b^{4}} \tilde{R}^{4}\right] .
$$

¿From Eqs. 4.43 and 4.45,

$$
\begin{aligned}
\Delta I= & I_{B H}-I_{A d S} \\
& =\frac{V_{3}}{16 \pi G_{5}}\left[\frac{2}{b^{2}} \tilde{R}^{4}\left(\beta-\beta^{\prime}\right)-\beta \frac{2}{b^{2}} r_{+}^{4}\right. \\
& \left.-\alpha^{\prime} \frac{5}{3 b^{4}}\left[\tilde{R}^{4}\left(\beta-\beta^{\prime}\right)-\beta r_{+}^{4}\right]-\alpha^{\prime} \beta \frac{3 \omega^{2} M^{2}}{r_{+}^{4}}\right] .
\end{aligned}
$$

As prescribed, we equate the boundary geometries at $r=\tilde{R}$ to obtain the relation between $\beta$ and $\beta^{\prime}$.

$$
\beta^{\prime}\left(1+\frac{\tilde{R}^{2}}{b^{2}}+\alpha^{\prime} \frac{\tilde{R}^{2}}{6 b^{4}}\right)^{1 / 2}=\beta\left(1+\frac{\tilde{R}^{2}}{b^{2}}-\frac{\omega M}{\tilde{R}^{2}}+\alpha^{\prime} \frac{\tilde{R}^{2}}{6 b^{4}}+\alpha^{\prime} \frac{M^{2} \omega^{2}}{2 \tilde{R}^{6}}\right)^{1 / 2} .
$$

Therefore,

$$
\beta^{\prime}=\beta\left(1-\frac{1}{2 \tilde{R}^{4}} \omega M b^{2}+\frac{\alpha^{\prime}}{12 \tilde{R}^{4}} \omega M\right) .
$$

Using this relation $\Delta I$ can be written as,

$$
\Delta I=-\frac{\beta V_{3}}{16 \pi G_{5}}\left[\frac{r_{+}^{4}}{b^{2}}-r_{+}^{2}+\frac{\alpha^{\prime}}{6 b^{4}}\left(36 b^{2} r_{+}^{2}+10 r_{+}^{4}+15 b^{4}\right)\right] .
$$

The energy of the black hole is then given by,

$$
\begin{aligned}
E & =\frac{\partial \Delta I}{\partial \beta} \\
& =\frac{V_{3}}{16 \pi G_{5}}\left[\frac{3}{b^{2}}\left(r_{+}^{4}+r_{+}^{2} b^{2}\right)-\frac{\alpha^{\prime}}{2 b^{4}} \frac{20 r_{+}^{6}+14 r_{+}^{4} b^{2}-6 r_{+}^{2} b^{4}-3 b^{6}}{b^{2}-2 r_{+}^{2}}\right] \\
& =M+O\left(\alpha^{\prime}\right) .
\end{aligned}
$$


And the Euclidean entropy works out to be,

$$
S=\frac{V_{3} r_{+}^{3}}{4 G_{5}}\left[1+\alpha^{\prime} \frac{2}{b^{2}}\left(1+\frac{3}{2} \frac{b^{2}}{r_{+}^{2}}\right)\right] .
$$

Note that though this is to leading order in $\alpha^{\prime}$, we have had to make no assumption on $\frac{r_{+}^{2}}{b^{2}}$. This answer disagrees with the expression Eq. 70 of [10]. But it agrees with the expressions found in [11].

Using Wald's formula 3.20 the expression for the entropy is

$$
S_{\text {Wald }}=\frac{1}{4 G_{5}} \int_{\text {Horizon }} d^{3} x \sqrt{h}\left[1+\frac{\alpha^{\prime}}{2}\left(R-2 h^{i j} R_{i j}+h^{i j} h^{k l} R_{i k j l}\right)\right] .
$$

Evaluating this expression to leading order in $\alpha^{\prime}$ gives the same answer Eq.4.51 as the Euclidean calculation.

\subsection{The $R^{4}$ Term}

Since one of our motivations to study the relation between the Euclidean and Wald expressions for entropy is the AdS/CFT correspondence, we should look at the corrections that appear in the Type IIB string effective action. The first non vanishing corrections involve eight derivatives - a term involving four powers of the Riemann tensor together with its supersymmetric counterparts.

In the context of corrections to $A d S$ black hole entropy, the $R^{4}$ term was studied in [12] ${ }^{9}$. They adopted the Euclidean approach and looked at the so called planar black hole (a large mass limit of the $A d S$ Schwarzschild solution $)^{10}$. They were able to compute the leading order correction to the entropy. Here we will study the correction using Wald's expression and obtain the same result as [12]. We have also further checked agreement with the finite mass results of $[24,25]$.

\section{- Review of the Euclidean Calculation}

The relevant pieces of the ten dimensional tree level type IIB superstring effective action are,

$$
\begin{gathered}
I=-\frac{1}{16 \pi G_{10}} \int d^{10} x \sqrt{-g_{10}}\left(R-\frac{1}{4.5 !}\left(F_{5}\right)^{2}\right)+I^{\prime}, \\
I^{\prime}=-\frac{\gamma}{16 \pi G_{10}} \int d^{10} x \sqrt{-g_{10}} W,
\end{gathered}
$$

\footnotetext{
${ }^{9}[23]$ discusses the effects of these higher derivative terms on the extremal D3 brane solution.

${ }^{10}$ This was generalised to the finite mass case in [24][25], who also used the Euclidean approach.
} 
where, $F_{5}$ is the self-dual five form field strength. Note that we have set the dilaton to a constant value $\phi_{0}$. Here,

$$
\gamma=\frac{1}{8} \zeta(3)\left(\alpha^{\prime}\right)^{3}
$$

and

$$
W=C^{h m n k} C_{p m n q} C_{h}{ }^{r s p} C_{r s k}^{q}+\frac{1}{2} C^{h k m n} C_{p q m n} C_{h}{ }^{r s p} C_{r s k}^{q} .
$$

where $C_{p q m n}$ is the Weyl tensor. To put the leading $R^{4}$ term in this form, one has to use the freedom of field redefinition of the metric.

We will consider $I^{\prime}$ as perturbation. The leading order Type IIB supergravity solution which we will study is the throat region of the non-extremal D3-brane.

$$
d s^{2}=\frac{r^{2}}{b^{2}}\left[-\left(1-\frac{r_{0}^{4}}{r^{4}}\right) d t^{2}+d \vec{x}^{2}\right]+\frac{b^{2}}{r^{2}}\left(1-\frac{r_{0}^{4}}{r^{4}}\right)^{-1} d r^{2}+b^{2} d \Omega_{5}^{2}
$$

together with a constant self-dual five form field strength having $N$ units of flux in the $S^{5}$ as well as the black hole spacetime. The radius $b$ is related to the ten dimensional parameters via,

$$
b^{4}=\frac{N \sqrt{2 G_{10}}}{\pi^{2}} .
$$

Including the eight derivative terms such as $I^{\prime}$, the ten dimensional solution Eq.4.57 gets corrected. However, for the leading order correction, we only need to take into account the term $I^{\prime}$. Moreover, it was argued in [12] (see also [26]) that one could compactify the ten dimensional action Eq.4.53 on the $S^{5}$ to get the effective five dimensional gravitational action, ${ }^{11}$

$$
I_{5}=-\frac{1}{16 \pi G_{5}} \int d^{5} x \sqrt{-g}\left[\left(R_{5}+\frac{12}{b^{4}}\right)+\gamma W\right]
$$

where,

$$
\frac{1}{16 \pi G_{5}}=\frac{\operatorname{Vol}\left(S^{5}\right)}{16 \pi G_{10}}=\frac{\pi^{3} b^{5}}{16 \pi G_{10}} .
$$

For the computation of the leading correction in $\gamma$ to the entropy, it suffices to consider this effective action and its solutions. It is consistent to set the dilaton to its constant value and take the five form to be self-dual.

\footnotetext{
${ }^{11}$ There are some caveats to be mentioned regarding the use of this five dimensional effective action. There can be higher derivative terms involving $F_{5}$ which might also contribute. See [23]. However, our point of view here is more limited and we are simply undertaking to check the equality between the computation of [12] done with this effective action, with Wald's approach. We thank K. Skenderis for discussion on this point.
} 
The five dimensional leading order solution ${ }^{12}$

$$
d s^{2}=\frac{r^{2}}{b^{2}}\left[-\left(1-\frac{r_{0}^{4}}{r^{4}}\right) d t^{2}+d \vec{x}^{2}\right]+\frac{b^{2}}{r^{2}}\left(1-\frac{r_{0}^{4}}{r^{4}}\right)^{-1} d r^{2} .
$$

can be obtained as a large mass limit of the metric Eq.2.2. The temperature is given by

$$
T=\frac{r_{0}}{\pi b^{2}}
$$

The leading entropy is

$$
S=\frac{V r_{0}^{3}}{4 b^{3} G_{5}}=\frac{\pi^{2}}{2} N^{2} V T^{3}
$$

where $V=\int d^{3} \vec{x}$ is the volume in the dual gauge theory.

The leading correction to the solution, Eq.4.61, from the Weyl term in Eq.4.59, is given by [12] (in terms of the functions entering the spherically symmetric ansatz Eq.4.5),

$$
\begin{gathered}
B(r)=\frac{r^{2}}{b^{2}}\left(1-\frac{r_{0}^{4}}{r^{4}}\right)\left[1-\frac{15 \gamma}{b^{6}} \frac{r_{0}^{4}}{r^{12}}\left(5 r^{8}+5 r^{4} r_{0}^{4}-3 r_{0}^{8}\right)\right] \\
A(r)=\frac{b^{2}}{r^{2}}\left(1-\frac{r_{0}^{4}}{r^{4}}\right)^{-1}\left[1+\frac{15 \gamma}{b^{6}} \frac{r_{0}^{4}}{r^{12}}\left(5 r^{8}+5 r^{4} r_{0}^{4}-19 r_{0}^{8}\right)\right] .
\end{gathered}
$$

One can then apply the Euclidean prescription as in the previous subsections. The final result is

$$
\begin{aligned}
S & =S_{0}\left(1+\frac{45 \gamma}{b^{6}}\right)+S_{0}\left(\frac{15 \gamma}{b^{6}}\right) \\
& =S_{0}\left(1+\frac{60 \gamma}{b^{6}}\right)
\end{aligned}
$$

where, $S_{0}$ is the Bekenstein-Hawking entropy evaluated on the area of the perturbed solution

$$
S_{0}=\frac{\text { Area }_{H}}{4 G_{5}}=\frac{V r_{0}^{3}}{4 b^{3} G_{5}} .
$$

The two terms in the first line come from the Einstein-Hilbert term and the Weyl term in the action respectively.

The correction to the temperature in the presence of the $R^{4}$ term is given by,

$$
T=\frac{r_{0}}{\pi b^{2}}\left(1+\frac{15 \gamma}{b^{2}}\right)
$$

\footnotetext{
${ }^{12}$ We will use $r_{0}$ for the location of the horizon of the planar black hole in keeping with [12], and use $r_{+}$ for that of the usual finite mass black hole.
} 
So the entropy given in Eq.4.63 is corrected to

$$
S=\frac{\pi^{2}}{2} N^{2} V T^{3}\left(1+\frac{15 \gamma}{b^{6}}\right)
$$

Since Wald's expression gives the area term from the Einstein-Hilbert Lagrangian, we will get $S_{0}$ after using the perturbed solution. Therefore what we will do is to calculate the correction to the entropy coming from the $R^{4}$ term using Wald's formula and show that the sum of the two contributions matches with the above result.

\section{- Wald's Formula for the $R^{4}$ Term}

We saw in Sec. 3.3 that the Wald expression for the entropy, for a Lagrangian which depends polynomially on the Riemann tensor, is given by

$$
S_{B H}=-2 \pi \int_{\mathcal{H}} d^{3} x \sqrt{h} \frac{\partial L}{\partial R_{\mu \nu \rho \sigma}} \epsilon_{\mu \nu} \epsilon_{\rho \sigma} .
$$

$\epsilon_{\mu \nu}$ is the binormal to the bifurcation surface, normalized such that $\epsilon_{\mu \nu} \epsilon^{\mu \nu}=-2$. We can take

$$
\epsilon_{\mu \nu}=\xi_{\mu} \eta_{\nu}-\xi_{\nu} \eta_{\mu}
$$

where $\xi$ and $\eta$ are null vectors normal to the bifurcate killing horizon, with $\xi \cdot \eta=1$. We will take $\xi$ to be the killing vector field,

$$
\xi=\frac{\partial}{\partial t}
$$

which is null at the bifurcate horizon. Then $\eta$ can be taken to be

$$
\eta=-f^{-1} \frac{\partial}{\partial t}-\frac{\partial}{\partial r}
$$

where $f=-\frac{r^{2}}{b^{2}}\left(1-\frac{r_{0}^{4}}{r^{4}}\right)$. In our particular case, we are interested in the additional contribution to the entropy from

$$
\Delta L=\frac{\gamma}{16 \pi G_{5}} W
$$

Therefore

$$
\Delta S=-\frac{\gamma}{8 G_{5}} \int_{\mathcal{H}} d^{3} x \sqrt{h} \frac{\partial W}{\partial R_{\mu \nu \rho \sigma}} \epsilon_{\mu \nu} \epsilon_{\rho \sigma} .
$$

Since $\mathrm{W}$ is function only of the Weyl tensor

$$
C_{a b c d}=R_{a b c d}+\frac{1}{3}\left(g_{a d} R_{c b}+g_{b c} R_{a d}-g_{a c} R_{d b}-g_{b d} R_{c a}\right)+\frac{1}{12}\left(g_{a c} g_{b d}-g_{a d} g_{c b}\right) R,
$$


we may write for $\Delta S$,

$$
\Delta S=-\frac{\gamma}{8 G_{5}} \int_{\mathcal{H}} d^{3} x \sqrt{h} \frac{\partial W}{\partial C_{a b c d}} \frac{\partial C_{a b c d}}{\partial R_{\mu \nu \rho \sigma}} \epsilon_{\mu \nu} \epsilon_{\rho \sigma} .
$$

We then have

$$
\frac{\partial W}{\partial C_{a b c d}}=W_{1}^{a b c d}+\frac{1}{2} W_{2}^{a b c d}
$$

where

$$
\begin{aligned}
W_{1}^{a b c d}= & g_{l i}\left[C_{s k j}^{d} C^{l b c j} C^{a k s i}+C_{j s k}^{d} C^{b l k c} C^{i j s a}\right. \\
& \left.+C_{j k s}^{d} C^{a j k l} C^{b s i c}+C_{k j s}^{d} C^{l j k a} C^{s b c i}\right]
\end{aligned}
$$

and

$$
\begin{aligned}
W_{2}^{a b c d}= & g_{l i}\left[C_{v k j}^{a} C^{i v k b} C^{j l c d}+C_{v k j}^{b} C^{i v k a} C^{l j c d}\right. \\
& \left.+C_{j k v}^{a} C^{d l k v} C^{i b c j}+C_{j k v}^{d} C^{l a v k} C^{j b c i}\right] .
\end{aligned}
$$

We will denote

$$
M_{a b c d}=\frac{\partial C_{a b c d}}{\partial R_{\mu \nu \rho \sigma}}\left(\xi_{\mu} \eta_{\nu}-\xi_{\nu} \eta_{\mu}\right)\left(\xi_{\rho} \eta_{\sigma}-\xi_{\sigma} \eta_{\rho}\right)
$$

where

$$
\begin{aligned}
\frac{\partial C_{a b c d}}{\partial R_{\mu \nu \rho \sigma}}= & \delta_{a}^{\mu} \delta_{b}^{\nu} \delta_{c}^{\rho} \delta_{d}^{\sigma} \\
& +\frac{1}{3}\left(g_{a d} g^{\mu \rho} \delta_{c}^{\nu} \delta_{b}^{\sigma}+g_{b c} g^{\mu \rho} \delta_{a}^{\nu} \delta_{d}^{\sigma}-g_{a c} g^{\mu \rho} \delta_{d}^{\nu} \delta_{b}^{\sigma}-g_{b d} g^{\mu \rho} \delta_{c}^{\nu} \delta_{a}^{\sigma}\right) \\
& +\frac{1}{24}\left(g_{a c} g_{b d}-g_{a d} g_{b c}\right)\left(g^{\mu \rho} g^{\nu \sigma}-g^{\mu \sigma} g^{\nu \rho}\right)
\end{aligned}
$$

Using the unperturbed metric Eq.4.61 one can calculate,

$$
W_{1}^{a b c d} M_{a b c d}=-\frac{232 r_{0}^{12}}{b^{6} r^{12}}
$$

and

$$
W_{2}^{a b c d} M_{a b c d}=\frac{224 r_{0}^{12}}{b^{6} r^{12}}
$$

Then the integrand in Eq. 4.76 becomes

$$
\left.\frac{\partial W}{\partial C_{a b c d}} \frac{\partial C_{a b c d}}{\partial R_{\mu \nu \rho \sigma}} \epsilon_{\mu \nu} \epsilon_{\rho \sigma}\right|_{r=r_{0}}=-\left.\frac{120 r_{0}^{12}}{b^{6} r^{12}}\right|_{r=r_{0}}=-\frac{120}{b^{6}} .
$$


Together with the factors of $\sqrt{h}=\frac{r_{0}^{3}}{b^{3}}$ and $V$ in the integral we get

$$
\Delta S=\frac{V r_{0}^{3}}{4 b^{3} G_{5}}\left(\frac{60 \gamma}{b^{6}}\right)=\frac{\pi^{2}}{2} N^{2} V T^{3}\left(\frac{60 \gamma}{b^{6}}\right)
$$

So the total entropy is,

$$
S=S_{0}+\Delta S=\frac{V r_{0}^{3}}{4 b^{3} G_{5}}\left(1+\frac{60 \gamma}{b^{6}}\right)=\frac{\pi^{2}}{2} N^{2} V T^{3}\left(1+\frac{15 \gamma}{b^{6}}\right)
$$

which agrees with Eqs.4.65,4.68. Note that the individual contributions from the EinsteinHilbert term and the Weyl term to the Euclidean entropy, given in the first line of Eq.4.65, are different from the individual contributions from these terms to the Wald entropy.

One can also look at the corrections to the AdS-Schwarzschild metric from the action 4.59 as in [25]. The Euclidean entropy in this case has been computed in [25] to be,

$$
S=\frac{V_{3} r_{+}^{3}}{4 G_{5}}\left[1+\frac{60 \gamma}{b^{6}}\left(1+\frac{b^{2}}{r_{+}^{2}}\right)^{3}\right] .
$$

In the Wald approach, the Einstein-Hilbert part of the action gives,

$$
S_{0}=\frac{V_{3} r_{+}^{3}}{4 G_{5}}
$$

The correction to the area law comes from the $R^{4}$ term. Following the same procedure as above can calculate the integrand in Eq. 4.76 using the unperturbed AdS-Schwarzschild metric. The final result is,

$$
\left.\frac{\partial W}{\partial C_{a b c d}} \frac{\partial C_{a b c d}}{\partial R_{\mu \nu \rho \sigma}} \epsilon_{\mu \nu} \epsilon_{\rho \sigma}\right|_{r=r_{+}}=-\frac{120 \gamma}{b^{6}}\left(1+\frac{b^{2}}{r_{+}^{2}}\right)^{3} .
$$

So the total entropy is given by Eq. 4.87 and once again the two approaches yield the same answer for any value of the mass.

\section{$5 \quad$ Wald's Approach Vs. Euclidean Approach in Asymp- totically AdS Spacetime}

Having seen that the computations in both approaches agree non-trivially in a number of examples, we have reason to be confident that this must generally be so. In this section, we will attempt to gain some understanding of why this might be so. We will start with looking at the Noetherian definition of mass in AdS spacetimes and see that it involves a subtraction procedure very like in the Euclidean framework. We will use this then, together with a line of argument due to Wald, made for the asymptotically flat case, to relate the Wald expression for the entropy to the Euclidean one. 


\subsection{Mass in asymptotically AdS spacetime}

There are several ways to define mass in asymptotically AdS spacetimes (see [13] for a comprehensive comparison and references). In [13] it was shown for the Einstein-Hilbert Lagrangian, with appropriate boundary conditions, that the Hamiltonian definition of Wald reviewed in Sec. 3.2 (see also [21]), agreed with several other definitions. In particular, we have from Eq.3.13,

$$
\delta H_{\xi}=\delta \int_{\tilde{R}} d S_{a b} \sqrt{-g} Q^{a b}[\xi] .
$$

The boundary term $\Theta$ that appears in Eq.3.13 does not contribute ${ }^{13}$. The Noether charge appearing in the right hand side is well defined in asymptotically AdS spacetimes only after introducing a cutoff at an outer boundary (at, say, $r=\tilde{R}$ ). The variation $\delta$ is then such that it keeps the geometry fixed at this hypersurface.

In the case, of the Einstein-Hilbert Lagrangian, it was shown in [13] that the boundary conditions allow for Eq.5.1 to be integrated to give a Hamiltonian $H_{\xi}$. The additive constant to the Hamiltonian is fixed by demanding that the energy is zero in pure AdS.

We will assume that the above Noetherian definition of mass continues to make sense for higher derivative Lagrangians of interest in asymptotically AdS spacetimes (reflected in appropriate boundary conditions). Namely, we will integrate Eq.5.1

$$
H_{\xi}=\left[\int_{\tilde{R}} d S_{a b} \sqrt{-g} Q^{a b}[\xi]-\int_{\tilde{R}} d S_{a b} \sqrt{-g} Q_{A d S}^{a b}[\tilde{\xi}]\right] .
$$

Again, the additive constant has been chosen so that the Hamiltonian is zero for pure AdS spacetime. Since it shouldn't contribute to the variation, the boundary geometry at $r=\tilde{R}$ must be the same for both the spacetime and the reference AdS. This implies that the killing vector field $\tilde{\xi}$ is normalised such that it agrees with that of $\xi$

$$
|\tilde{\xi}|^{2}=|\xi|^{2} .
$$

on the boundary hypersurface.

\footnotetext{
${ }^{13}$ For simplicity, we will assume in what follows that the boundary term always cancels out on subtraction from the background. Which is why we have dropped it in Eq.5.2. This is true for the Einstein-Hilbert Lagrangian and we have checked that it also holds for the Gauss-Bonnet case. We believe this must be a general feature in the asymptotically AdS case. This is unlike the flat space case where the boundary term is crucial to the Euclidean computation, being the only contribution to the mass. In any case, even if a boundary term contributes, the argument below can be modified by including the corresponding term in the Euclidean computation of the action as well.
} 
Since $Q[\xi]$ is linear in $\xi$ by construction from Eq.3.6, and since the difference in normalisation between $\xi$ and $\tilde{\xi}$ is a constant, we can write,

$$
Q_{A d S}^{a b}[\tilde{\xi}]=\left[\left(\frac{g_{t t}}{g_{t t}^{A d S}}\right)^{\frac{1}{2}}\right]_{r=\tilde{R}} Q_{A d S}^{a b}[\xi] .
$$

Here we have taken $\xi$ to be the time translation killing vector. So,

$$
\int_{\tilde{R}} d S_{a b} \sqrt{-g} Q_{A d S}^{a b}[\tilde{\xi}]=\left[\left(\frac{g_{t t}}{g_{t t}^{A d S}}\right)^{\frac{1}{2}}\right]_{r=\tilde{R}} \int_{\tilde{R}} d S_{a b} \sqrt{-g} Q_{A d S}^{a b}[\xi] .
$$

The Hamiltonian which is the total energy or mass of the system is then given by

$$
\mathcal{E}=\left[\int_{\tilde{R}} d S_{a b} \sqrt{-g} Q^{a b}[t]-\left[\left(\frac{g_{t t}}{g_{t t}^{A d S}}\right)^{\frac{1}{2}}\right]_{r=\tilde{R}} \int_{\tilde{R}} d S_{a b} \sqrt{-g} Q_{A d S}^{a b}[t]\right] .
$$

It is clear from the above expression that for pure AdS space time, $g_{t t}=g_{t t}^{A d S}$, and hence $H_{\xi}=0$.

In the Appendix we explicitly calculate the energy of the AdS Schwarzschild metric Eq.2.2 using this prescription. We see there that the subtraction plays exactly the same role as it did in the Euclidean computation. It is necessary for correctly getting the finite answer.

\subsection{Relating the Wald and Euclidean approaches}

Using the above definition of mass, we can make a direct connection between the Wald and Euclidean approaches by modifying an argument given by Wald [4] for the asymptotically flat case.

As in Sec.3.3 we will consider $\xi$ to be a killing vector vanishing on the bifurcate horizon. In that case $\mathcal{L}_{\xi} \psi=0$ and hence $\boldsymbol{\Theta}\left(\mathcal{L}_{\xi} \psi\right)=0$. Therefore the Noether current simplifies to

$$
\mathbf{J}=-\xi \cdot \mathbf{L}
$$

Integrating both sides of Eq.5.7 over a constant time hypersurface $\mathcal{C}$ of the black hole spacetime, having the interior boundary $\mathcal{H}$ and the outer boundary at $r=\tilde{R}$. We get

$$
\begin{gathered}
\int_{\mathcal{C}} d V_{t} J^{t}=-\int_{\mathcal{C}} d V_{t} \xi^{t} L\left(g_{B H}\right) \\
-\int_{\mathcal{H}} d S_{a b} \sqrt{-g} Q^{a b}+\int_{\tilde{R}} d S_{a b} \sqrt{-g} Q^{a b}=-\int_{\mathcal{C}} d V_{t} \xi^{t} L\left(g_{B H}\right) .
\end{gathered}
$$


Therefore

$$
\int_{\mathcal{H}} d S_{a b} \sqrt{-g} Q^{a b}\left[\xi^{t}\right]=\mathcal{E}+\int_{\mathcal{C}} d V_{t} \xi^{t} L\left(g_{B H}\right)+\left[\left(\frac{g_{t t}^{B H}}{g_{t t}^{A d S}}\right)^{\frac{1}{2}}\right]_{r=\tilde{R}} \int_{\tilde{R}} d S_{a b} \sqrt{-g} Q_{A d S}^{a b}\left[\xi^{t}\right] .
$$

Using the same logic as above, but now integrating over $\Sigma$ a Cauchy hypersurface in global AdS, we have

$$
\int_{\tilde{R}} d S_{a b} \sqrt{-g} Q_{A d S}^{a b}\left[\xi^{t}\right]=-\int_{\Sigma} d V_{t} \xi^{t} L\left(g_{A d S}\right)
$$

As a result,

$$
\int_{\mathcal{H}} d S_{a b} \sqrt{-g} Q^{a b}=\mathcal{E}+\int_{\mathcal{C}} d V_{t} \xi^{t} L\left(g_{B H}\right)-\left(\frac{g_{t t}}{g_{t t}^{A d S}}\right)^{\frac{1}{2}} \int_{\Sigma} d V_{t} \xi^{t} L_{A d S}
$$

Since the Wald entropy of the black hole is

$$
S=\beta \int_{\mathcal{H}} d S_{a b} \sqrt{-g} Q^{a b}
$$

Eq.5.10 becomes

$$
S=\beta \mathcal{E}+\beta \int_{\mathcal{C}} d V_{t} \xi^{t} L\left(g_{B H}\right)-\beta\left(\frac{g_{t t}}{g_{t t}^{A d S}}\right)^{\frac{1}{2}} \int_{\mathcal{C}} d V_{t} \xi^{t} L\left(g_{A d S}\right)
$$

Since, we have a static background

$$
I_{B H}=-\beta \int_{\Sigma} d V_{t} \xi^{t} L\left(g_{B H}\right)
$$

and

$$
I_{A d S}=-\beta\left(\frac{g_{t t}}{g_{t t}^{A d S}}\right)^{\frac{1}{2}} \int_{\mathcal{C}} d V_{t} \xi^{t} L\left(g_{A d S}\right)
$$

provided we assign a temperature $\beta^{\prime}$ to $A d S$ space which is

$$
\beta^{\prime}=\beta\left(\frac{g_{t t}}{g_{t t}^{A d S}}\right)^{\frac{1}{2}}
$$

We now see exactly the Euclidean prescription, where one subtracts the action of a background AdS spacetime with the above identification of temperatures. In other words,

$$
S=\beta \mathcal{E}-I_{B H}+I_{A d S}=\beta \mathcal{E}-\Delta I .
$$

Thus starting from the Noetherian expressions for the entropy, we obtain the relation to the Euclidean prescription with exactly the same subtraction procedure. ${ }^{14}$

Though the mass that appears above is the Noetherian definition of mass, it must be that it agrees with the Euclidean prescription for the mass. This is because both Euclidean and Noetherian prescriptions obey the first law. In appendix B we indicate the argument.

\footnotetext{
14 [14] derives this relation for the general two derivative lagrangian in asymptotically locally $A d S$ spaces. See also footnote 3 .
} 


\section{Conclusions}

We have studied the relation between the Euclidean and Noetherian approaches to the entropy of asymptotically $A d S$ black holes with the aim of shedding light on the two holographic descriptions for black hole entropy. The agreement between these two approaches can be understood, as we have described, from the general construction of Noether charges. The explicit computations in a number of examples further bolsters the case for the equivalence of the two approaches.

What would be nice to see is if the argument for this equivalence can be cast in a way which makes the relation of the Wald's formula to the gauge theory more transparent. It would help us answer the question: What is the meaning of Wald's formula in the dual Gauge Theory? Given the generality of Wald's formula, it seems likely that there is an equally universal statement to be made in the dual gauge theory. It has presumably to do with the behaviour of the number of degrees of freedom under $R G$ flow in the gauge theory. The boundary holographic description (which is related to the Euclidean approach) is naturally an expansion about small gauge coupling, moving inwards from the UV so to say. While the Wald expression is an expansion in inverse powers of the gauge coupling, systematically moving outwards from the IR. In this context, perhaps a generalisation of the entropy function of Sen $[27,28]$ might be a useful way to understand the interpolation.

Another point which is worth noting from the explicit results we have exhibited is the relative computational simplicity of the Wald approach in evaluating corrections. It seems

to be less onerous than the Euclidean procedure. This is also a sign that the Wald approach is more natural, at least for large gauge coupling.

\section{Acknowledgment}

We would like to thank D. Astefanesei and especially A. Sen for helpful comments during the course of this work. We also wish to thank K. Skenderis for very useful correspondence. We are also indebted to the people of India for their support. 


\section{Appendix}

\section{A Calculation of Energy}

Let us calculate the total energy for the AdS-Schwarzschild space time

$$
d s^{2}=-\left(1+\frac{r^{2}}{b^{2}}-\frac{\omega M}{r^{2}}\right) d t^{2}+\left(1+\frac{r^{2}}{b^{2}}-\frac{\omega M}{r^{2}}\right)^{-1} d r^{2}+r^{2} d \Omega_{3}^{2}
$$

with the Einstein-Hilbert Lagrangian

$$
L=\frac{1}{16 \pi G_{5}}\left(R+\frac{12}{b^{2}}\right) .
$$

For this Lagrangian,

$$
Q^{a b}=-\frac{1}{16 \pi G_{5}}\left(\nabla^{a} \xi^{b}-\nabla^{b} \xi^{a}\right) .
$$

Let $\xi$ be an asymptotic time translational vector,

$$
\xi=\frac{\partial}{\partial t}, \quad \xi^{t}=1
$$

So

$$
Q^{t r}=\frac{1}{16 \pi G_{5}}\left(\partial_{r} g_{t t}\right) \xi^{t}
$$

For the AdS-Schwarzschild metric,

$$
Q^{t r}=\frac{2}{16 \pi G_{5}}\left(\frac{r}{b^{2}}+\frac{\omega M}{r^{3}}\right) .
$$

The Noetherian definition of mass Eq.5.6 is

$$
\mathcal{E}=\left[\int_{\tilde{R}} d S_{a b} \sqrt{-g} Q^{a b}[t]-\int_{\tilde{R}} d S_{a b} \sqrt{-g} Q_{A d S}^{a b}[\tilde{t}]\right] .
$$

Now,

$$
\int_{\tilde{R}} d S_{a b} \sqrt{-g} Q^{a b}[t]=\int_{\tilde{R}} d \mathbf{S}_{\mathbf{t r}} Q^{t r}[t]
$$

where $\mathbf{d S}^{\mathbf{a b}}=\frac{1}{2}\left(d x^{a} \otimes d x^{b}-d x^{b} \otimes d x^{a}\right) \sqrt{h}$ is the volume element on the boundary. So putting every thing together we get,

$$
\int_{\tilde{R}} d S_{a b} \sqrt{-g} Q^{a b}[t]=\frac{2 V}{16 \pi G_{5}}\left(\frac{\tilde{R}^{4}}{b^{2}}+\omega M\right)=\frac{2 V}{16 \pi G_{5}} \frac{\tilde{R}^{4}}{b^{2}}+\frac{2 M}{3} .
$$


Let us calculate the contribution from the background AdS metric,

$$
d s_{A d S}^{2}=-\left(1+\frac{r^{2}}{b^{2}}\right) d t^{2}+\left(1+\frac{r^{2}}{b^{2}}\right)^{-1} d r^{2}+r^{2} d \Omega_{3}^{2}
$$

Here

$$
Q_{A d S}^{t r}=\frac{2}{16 \pi G_{5}} \frac{r}{b^{2}} \tilde{\xi}^{t}
$$

where the asymptotic time translation vector $\tilde{\xi}=\tilde{\xi}^{t} \frac{\partial}{\partial t}$ in background AdS space is given by,

$$
\tilde{\xi}^{t}=\left(\frac{g_{t t}}{g_{t t}^{A d S}}\right)^{\frac{1}{2}}=1-\frac{\omega M b^{2}}{2 \tilde{R}^{4}}
$$

Therefore

$$
\int_{\tilde{R}} d S_{a b} \sqrt{-g} Q_{A d S}^{a b}\left[\tilde{\xi}^{t}\right]=\frac{2 V}{16 \pi G_{5}} \frac{\tilde{R}^{4}}{b^{2}}\left(1-\frac{\omega M b^{2}}{2 \tilde{R}^{4}}\right)=\frac{2 V}{16 \pi G_{5}} \frac{\tilde{R}^{4}}{b^{2}}-\frac{M}{3} .
$$

This finally implies

$$
\mathcal{E}=M
$$

\section{B Relating the Noetherian and Euclidean definitions of Mass}

In this section we will show that the Euclidean definition of mass is the same as that given by the Noetherian method. The Euclidean definition of mass is given by,

$$
M=\frac{\partial \Delta I}{\partial \beta}
$$

where $\Delta I=I_{B H}-I_{A d S}$,

$$
I_{B H}=-\beta \int_{\mathcal{C}} \xi \cdot \mathbf{L}, \quad I_{A d S}=-\beta_{A d S} \int_{\Sigma} \xi \cdot \mathbf{L}_{\mathbf{A d S}} .
$$

Using Eq.5.7, $I_{B H}$ and $I_{A d S}$ can be written as,

$$
I_{B H}=\beta \int_{\tilde{R}} Q[\xi]-\beta \int_{\mathcal{H}} Q[\xi]
$$

and

$$
I_{A d S}=\beta\left(\frac{g_{t t}}{g_{t t}^{A d S}}\right)^{1 / 2} \int_{\tilde{R}} Q_{A d S}[\xi]
$$


So,

$$
\begin{gathered}
\frac{\partial \Delta I}{\partial \beta}=\int_{\tilde{R}} Q[\xi]-\left(\frac{g_{t t}}{g_{t t}^{A d S}}\right)^{1 / 2} \int_{\tilde{R}} Q_{A d S}[\xi]-\frac{\partial}{\partial \beta}\left(\beta \int_{\mathcal{H}} Q[\xi]\right)+\beta \frac{\partial \mathcal{E}}{\partial \beta} \\
=\mathcal{E}-\left[\frac{\partial}{\partial \beta}\left(\beta \int_{\mathcal{H}} Q[\xi]\right)-\beta \frac{\partial \mathcal{E}}{\partial \beta}\right]
\end{gathered}
$$

In the first line (as well as to go to the second line) we have used the Noetherian definition of mass Eq.5.6. Since the Wald's expression for the entropy obeys the first law, the term inside the bracket vanishes. We therefore get

$$
\frac{\partial \Delta I}{\partial \beta}=\mathcal{E} .
$$

\section{References}

[1] J. M. Maldacena, "The large N limit of superconformal field theories and supergravity," Adv. Theor. Math. Phys. 2, 231 (1998) [Int. J. Theor. Phys. 38, 1113 (1999)] [arXiv:hepth/9711200].

[2] S. S. Gubser, I. R. Klebanov and A. W. Peet, "Entropy and Temperature of Black 3-Branes," Phys. Rev. D 54, 3915 (1996) [arXiv:hep-th/9602135].

[3] E. Witten, "Anti-de Sitter space, thermal phase transition, and confinement in gauge theories," Adv. Theor. Math. Phys. 2, 505 (1998) [arXiv:hep-th/9803131].

[4] R. M. Wald, "Black hole entropy in the Noether charge," Phys. Rev. D 48, 3427 (1993) [arXiv:gr-qc/9307038].

[5] S. W. Hawking and D. N. Page, "Thermodynamics Of Black Holes In Anti-De Sitter Space," Commun. Math. Phys. 87, 577 (1983).

[6] P. Kraus and F. Larsen, "Microscopic black hole entropy in theories with higher derivatives," JHEP 0509, 034 (2005) [arXiv:hep-th/0506176].

P. Kraus and F. Larsen, "Holographic gravitational anomalies," JHEP 0601, 022 (2006) [arXiv:hep-th/0508218].

[7] B. Sahoo and A. Sen, "BTZ black hole with Chern-Simons and higher derivative terms," arXiv:hep-th/0601228. 
[8] B. Sahoo and A. Sen, "Higher derivative corrections to non-supersymmetric extremal black holes in $\mathrm{N}=2$ supergravity," arXiv:hep-th/0603149.

[9] R. G. Cai, "Gauss-Bonnet black holes in AdS spaces," Phys. Rev. D 65, 084014 (2002) [arXiv:hep-th/0109133].

M. Cvetic, S. Nojiri and S. D. Odintsov, "Black hole thermodynamics and negative entropy in deSitter and Nucl. Phys. B 628, 295 (2002) [arXiv:hep-th/0112045].

I. P. Neupane, "Black hole entropy in string-generated gravity models," Phys. Rev. D 67, 061501 (2003) [arXiv:hep-th/0212092].

I. P. Neupane, "Thermodynamic and gravitational instability on hyperbolic spaces," Phys. Rev. D 69, 084011 (2004) [arXiv:hep-th/0302132].

[10] S. Nojiri, S. D. Odintsov and S. Ogushi, "Cosmological and black hole brane world universes in higher derivative gravity," Phys. Rev. D 65, 023521 (2002) [arXiv:hepth/0108172].

S. Nojiri and S. D. Odintsov, "Anti-de Sitter black hole thermodynamics in higher derivative gravity and new confining-deconfining phases in dual CFT," Phys. Lett. B 521, 87 (2001) [Erratum-ibid. B 542, 301 (2002)] [arXiv:hep-th/0109122].

[11] Y. M. Cho and I. P. Neupane, "Anti-de Sitter black holes, thermal phase transition and holography in higher curvature gravity," Phys. Rev. D 66, 024044 (2002) [arXiv:hepth/0202140].

[12] S. S. Gubser, I. R. Klebanov and A. A. Tseytlin, "Coupling constant dependence in the thermodynamics of $\mathrm{N}=4$ supersymmetric Yang-Mills theory," Nucl. Phys. B 534, 202 (1998) [arXiv:hep-th/9805156].

[13] S. Hollands, A. Ishibashi and D. Marolf, "Comparison between various notions of conserved charges in asymptotically AdS-spacetimes," Class. Quant. Grav. 22, 2881 (2005) [arXiv:hep-th/0503045].

[14] I. Papadimitriou and K. Skenderis, "Thermodynamics of asymptotically locally AdS spacetimes," JHEP 0508, 004 (2005) [arXiv:hep-th/0505190].

[15] N. Okuyama and J. i. Koga, "Asymptotically anti de Sitter spacetimes and conserved quantities in higher curvature gravitational theories," Phys. Rev. D 71, 084009 (2005) [arXiv:hep-th/0501044]. 
[16] J. i. Koga, "The first law of AdS black holes in higher curvature gravity," Phys. Rev. D 72, 064010 (2005) [arXiv:hep-th/0505219].

[17] D. V. Fursaev and S. N. Solodukhin, "On one loop renormalization of black hole entropy," Phys. Lett. B 365, 51 (1996) [arXiv:hep-th/9412020].

D. V. Fursaev and S. N. Solodukhin, Phys. Rev. D 52, 2133 (1995) [arXiv:hepth/9501127].

V. P. Frolov, W. Israel and S. N. Solodukhin, "On One-loop Quantum Corrections to the Thermodynamics of Charged Black Holes," Phys. Rev. D 54, 2732 (1996) [arXiv:hepth/9602105].

[18] G. W. Gibbons and S. W. Hawking, "Action Integrals And Partition Functions In Quantum Gravity," Phys. Rev. D 15, 2752 (1977).

[19] V. Iyer and R. M. Wald, "Some properties of Noether charge and a proposal for dynamical black hole entropy," Phys. Rev. D 50, 846 (1994) [arXiv:gr-qc/9403028].

[20] J. Lee and R. M. Wald, "Local Symmetries And Constraints," J. Math. Phys. 31 (1990) 725.

[21] R. M. Wald and A. Zoupas, "A General Definition of "Conserved Quantities" in General Relativity and Other Theories of Gravity," Phys. Rev. D 61, 084027 (2000) [arXiv:grqc/9911095].

[22] R. C. Myers, "Black holes in higher curvature gravity," arXiv:gr-qc/9811042.

[23] S. de Haro, A. Sinkovics and K. Skenderis, "On alpha' corrections to D-brane solutions," Phys. Rev. D 68, 066001 (2003) [arXiv:hep-th/0302136].

S. de Haro, A. Sinkovics and K. Skenderis, Phys. Rev. D 67, 084010 (2003) [arXiv:hepth/0210080].

[24] Y. h. Gao and M. Li, "Large N strong/weak coupling phase transition and the correspondence principle," Nucl. Phys. B 551, 229 (1999) [arXiv:hep-th/9810053].

[25] K. Landsteiner, "String corrections to the Hawking-page phase transition," Mod. Phys. Lett. A 14, 379 (1999) [arXiv:hep-th/9901143].

[26] J. Pawelczyk and S. Theisen, "AdS(5) x S(5) black hole metric at O(alpha'**3)," JHEP 9809, 010 (1998) [arXiv:hep-th/9808126]. 
[27] A. Sen, "Entropy function for heterotic black holes," arXiv:hep-th/0508042.

[28] A. Sen, "Black hole entropy function and the attractor mechanism in higher derivative gravity," JHEP 0509, 038 (2005) [arXiv:hep-th/0506177].

[29] E. Witten, "Anti-de Sitter space and holography," Adv. Theor. Math. Phys. 2, 253 (1998) [arXiv:hep-th/9802150]. 